Naḥw al-qulūb al-ṣagìir : La " grammaire des cœurs » de 'Abd al-Karīm al-Qušayrī

Présentation et traduction annotée

\title{
Francesco Chiabotti
}

\section{OpenEdition}

\section{Journals}

Édition électronique

URL : http://journals.openedition.org/beo/83

DOI : 10.4000/beo.83

ISBN : 978-2-35159-316-5

ISSN : 2077-4079

\section{Éditeur}

Presses de l'Institut français du Proche-Orient

\section{Édition imprimée}

Date de publication : 1 septembre 2009

Pagination : 385-402

ISBN : 978-2-35159-143-7

ISSN : 0253-1623

\section{Référence électronique}

Francesco Chiabotti, « Naḥw al-qulūb al-șagìr : La " grammaire des cœurs » de 'Abd al-Karīm alQušayrī », Bulletin d'études orientales [En ligne], Tome LVIII I Septembre 2009, mis en ligne le 01 septembre 2010, consulté le 30 avril 2019. URL : http://journals.openedition.org/beo/83 ; DOI : 10.4000/beo.83 


\section{NAHW AL-QULŪB AL-ȘAĠITR : LA « GRAMMAIRE DES CEEURS » DE 'ABD AL-KARĪM AL- QUŠ́AYRI \\ Présentation et traduction annotée}

Francesco CHIABOTTI

Doctorant à l'Université de Provence

"Il est inutile de scruter les œuvres des mystiques musulmans si l'on n'étudie pas de très près le mécanisme de la grammaire arabe, lexicographie, morphologie et syntaxe. Ces auteurs rattachent constamment les termes techniques qu'il proposent à leurs valeurs ordinaires, à l'usage courant constaté par les grammairiens".

Louis Massignon $^{1}$

\section{A. LE MÉTALANGAGE DE LA GRAMMAIRE ARABE.}

M.G. Carter, dans son article sur les origines de la grammaire arabe ${ }^{2}$, s'interroge sur l'origine du lexique technique de la grammaire. Les grammairiens arabes, dans la recherche d'un double langage technique capable de rendre les «objets » linguistiques ainsi que leur relations, ont opté pour un métalangage de surprenante simplicité, qui ne montre pas toujours un lien logique avec la réalité linguistique qu'il est censé décrire ${ }^{3}$. Carter note aussi un autre élément caractéristique de la langue arabe, reflet possible d'une arabica forma mentis: le rapprochement entre comportement humain et le mouvement le long d'une ligne, d'une direction. Šarîa, șirāt mustaqìm, țarīqa, sìra, madhab, dalīl, sont tous des termes techniques tirés de la notion de voie, de chemin. Et nahw ne fait pas exception. Carter conclut que «loin d'être l'expression de principes logiques, le langage est pour Sïbawayh exactement son contraire : une forme de comportement humain ${ }^{4}$. Le langage, dans sa terminologie, subit une personnification qui montre encore une fois l'attitude des anciens philologues arabes devant le fait linguistique. Carter propose donc un passage de

1. Cité par Michel Allard, Le problème des attributs divins dans la doctrine de al-Ash'ari et de ses premiers grand disciples, Imprimerie Catholique, Beyrouth, 1965, p. 2-3.

2. M.G. CARTER, « Les origines de la grammaire arabe », Revue des Etudes Islamique 40 (Paris, 1972), p. 69-97. [Trad. anglaise : "The beginnings of Arabic grammar », in The Early Islamic Grammatical Tradition, ed. par Ramzi Baalbaki. (The Formation of Classical Islamic World, v. 36), Ashgate Publishing Limited, 2007, p. 1-27. ]

3. M.G. CARTER, op. cit., p. 80

4. M.G. CARTER, op. cit., p. 82. 
lecture de ce lexique technique, tel qu'on le retrouve dans le Kitāb de Sïbawayh. Il parle de la «métaphore sociale » du langage ${ }^{5}$ : le grand grammairien d'origine persane aurait "personnifié » la terminologie technique de la grammaire parce qu'il prenait, pour créer son système, le modèle comportemental même de la société.

Kees Versteegh partage sur ce point le même avis que Carter, lorsqu'il écrit que les grammairiens arabes décrivent les éléments du langage comme dans une relation de force/ faiblesse. Leur terminologie décrit les faits linguistiques sous la forme d'une société de mots, une société caractérisée par une compétition entre éléments forts et éléments faibles. La force dans le système linguistique implique des « droits » d'un élément et son pouvoir sur les autres. Le langage est donc analysé comme la société humaine, fondée sur des relations de force entre ses composantes. Ce passage est possible selon Versteegh parce que les anciens philologues regardaient la grammaire comme une structure cohérente, dont les arguments pouvaient être appliqués en croisant les catégories et les éléments : une ressemblance dans une partie de la structure peut être utilisée pour expliquer une autre partie de la structure. Considérant la cohérence structurelle de la création, les savants islamiques n'ont pas vu d'objection au fait d'emprunter des arguments tirés des sciences exactes - ou sociales pour expliquer des phénomènes linguistiques. Le langage fait partie de la création et obéit selon ce principe aux mêmes lois qui la régissent ${ }^{6}$. En plus de la terminologie éthique (comme les termes qui décrivent le degré d'exactitude d'une affirmation ou de correction d'un comportement : hasan, qabīh, mustaqìm, muhāal), Sībawayh utilise des termes d'origine légale : qiyās, hiyār, hadd, etc. Le langage, métaphore très concrète d'une société, reflète aussi la loi qui la régit : les questions juridiques ont comme fondement une compréhension exacte des textes normatifs. Dans le Naḥw al-qulūb de Qušayrī, on perçoit la continuité de cet esprit ancien qui justifie le passage d'une discipline à l'autre. L'apparat des règles qui gère la langue, sans être le même de celui de l'âme, peut être appliqué à cette dernière selon une loi de relation analogique et d'harmonie qui s'étend à toute la création. L'effort de Qušayrī va justement dans ce sens : sortir la grammaire de son particularisme technique pour la ramener à sa portée universelle.

\section{B. LA NAISSANCE DU LANGAGE TECHNIQUE DE LA GRAMMAIRE ET DE LA MYSTIQUE: LE LANGAGE COMME CLÉ HERMÉNEUTIQUE DE LA RÉVÉLATION DANS LE TAFSĪR DE MUQĀTIL (M. 150/767).}

L'accès à la compréhension du texte révélé est passé dans ses premières époques surtout par la compréhension de son lexique. Selon Kees Versteegh, la grammaire comme science est issue de l'intérêt des premiers savants islamiques pour le texte coranique. La codification du Coran a impliqué un travail fondamentalement philologique, qui touchait la réforme de l'orthographe, l'établissement des variantes de lecture, l'explication des formes

5. «La reconnaissance de la métaphore sociale est la clé qui permet de comprendre le Kitāb, dont les critères et les méthodes ne sont qu'un prolongement de ceux de la morale et du droit. », M.G. Carter op. cit., p. 83.

6. Kees VeRSTEEGH, « The development of linguistic theory: Az-Zajjajjī on linguistic explanation », in Landmarks in linguistic though III. The Arabic linguistic tradition, London - New York, 1997, p. 73. 
linguistiques difficiles. La lexicographie est peut-être la première science du langage à se développer au sein de l'exégèse ${ }^{7}$.

Ce qui nous intéresse ici, c'est de remarquer comment l'herméneutique, d'un côté a pu aider à la constitution de la terminologie grammaticale, de l'autre est à l'origine du langage technique de la mystique. Cette observation est centrale dans la présente recherche pour mieux situer la rencontre entre grammaire et mystique que propose Qušayrī. Dans l'impossibilité de tracer la préhistoire de cette relation qui, dans l'état actuel de nos connaissances paraît avoir être inaugurée par Qušayrī, on se limitera à remarquer que la phase ancienne de l'exégèse est aussi une des premières sources du langage mystique. Certes P. Nwyia a fait déjà le tour de la question et on peut remarquer qu'au premier stade de son étude, on retrouve le tafsìr de Muqātil ${ }^{8}$. La naissance de la terminologie grammaticale et de la terminologie soufie se trouveraient donc rassemblée dans le tafsìr de Muqātil. Sans être ni philologue ni mystique, ce personnage nous indique l'ancienneté du lien qu'on essaye ici de tracer.

Le Naḥw al-qulūb de Qušayrī vise une intériorisation des notions de la grammaire. Dans ce sens, si d'un côté l'exégèse s'est développée a partir du Coran dans une direction qui reste extérieure - philologique, historique, juridique -, de l'autre, grâce à l'approfondissement de la lecture soufie, la terminologie coranique à été interprété dans uns sens large, lié au un processus de l'expérience directe du croyant :

«La reprise de l'œuvre de Muqātil par Tirmidhī montre comment s'est fait le passage d'un vocabulaire à un autre par l'enrichissement qu'apporte l'expérience du texte coranique. $»^{9}$

Avec le Nahw al-qulūb, on se trouve dans une démarche comparable. Certes, le vocabulaire dont Nwyia parle est coranique, alors que Qušayrī essaye d'opérer un passage du vocabulaire technique de la grammaire à celui - technique lui aussi, mais sur une autre forme - du soufisme. La clé qui permet ce passage, et sur la quelle Qušayrī dans ses écrits insiste fortement, c'est justement la notion d' «expérimentation interne ${ }^{10}$. Le Nahw alqulüb est un exemple significatif de l'effort de Qušayrî pour établir un pont entre les sciences islamique et le soufisme. La relation entre savoir des 'ulamā' et expérience mystique, dans l'œuvre de Qušayrī, n'est pas neutre. Dans la Wașiya li-l-murīdin ${ }^{11}$ il explique que se lier à un autre chemin que celui des soufis, ne permet pas de réaliser le parcours spirituel :

«Les gens sont soit partisans de la transmission et de la tradition (așhāb al-naql wa al-atar) ou partisans de l'intellect et de la réflexion (așhāa bl-'aql wa al-fikr). Les maitres de ce groupe [le soufisme] sont au-dessus de tout cela. Ce qui est caché aux autres hommes,

\footnotetext{
7. Kees VersteEgh, «Linguistic and exegesis : Muqātil on the explanation of the Qur'ān », in Landmarks in linguistic though III. The Arabic linguistic tradition, London - New York, 1997, p. 11-23.

8. P. NWYIA, Langage mystique et exégèse coranique, Beyrouth, 1970, p. 24-25.

9. P. NWYIA, op. cit., p. 156.

10. P. NWYIA, op. cit., p. 157.

11. Chapitre conclusif de la Risāla. (Al-Risāla al-Qušayriyya, éd. par 'Abd al-Ḥalīm Maḥmūd, Damas, Dār al-ḩayr, 2003).
} 
est pour eux manifeste, et ce que les gens désirent atteindre par la connaissance, eux le tiennent de Dieu ${ }^{12}$.»

Le Naḥw al-qulüb est cohérent avec cette perspective proposée dans la Risāla et en représente une actualisation concrète. L'analyse du texte montre aussi une large convergence terminologique entre les deux textes. La grammaire des cœurs s'oppose à la grammaire des intellects. Qušayrī montre le danger d'une science privée d'esprit. Cet esprit est le fruit d'une éducation spirituelle dont le Naḥw al-qulūb est aussi la description. Dans ce texte, l'engagement de Qušayrī dans la revivification de l'expérience spirituelle directe et personnelle apparaît évident. Il s'oppose à un savoir livresque, purement littéral, transmis par les savants des sciences extérieures et qui n'engage pas l'être dans une véritable transformation de soi. Les sciences extérieures, dans la perspective de Qušayrī, sont les garants de cette expérience, mais elles n'épuisent pas l'intégralité de la connaissance. La grammaire est donc prise à titre d'exemple d'un savoir qui peut demeurer superficiel, formel, ou au contraire devenir une source de connaissance pour exprimer les expériences du cheminement spirituel. Le Naḥw al-qulüb est basée sur la notion d'allusion au sens caché, la išāra. Qušayrī cherche une signification ultérieure à la terminologie grammaticale, en l'exploitant d'un point de vue initiatique. La description des éléments du langage est rapprochée de certains aspects de la doctrine soufie sur les états de l'âme et sur le cheminement initiatique. Le passage est effectué à partir de la notion de išāra, l'allusion spirituelle. P. Nwyia, dans son article dans la $\mathrm{EI}^{2}$ a parcouru l'histoire de ce terme dans le soufisme :

«Quand les Sūfīs s'appellent les ahl al-išāra (école allusioniste) ou quand ils disent que leurs sciences sont des 'ulūm al-išăra, ils entendent par là définir non seulement leur mode de s'exprimer, mais aussi le contenu d'une expérience qui ne peut être évoqué qu'à travers ce mode. ${ }^{13}$

Pour Qušayrī le langage allusif parle seulement à ceux qui ont déjà fait l'expérience des réalités véhiculées par le discours. Les allusions sont donc des moyens pour indiquer des réalités spirituelles sans les décrire ou les nommer ouvertement. L'utilisation de l'išăra marque une convergence très significative entre cet ouvrage "mineur » de Qušayrī et son commentaire soufi, les Lațā̉if al-išārāt. La recherche des sens allusifs tirés des versets coraniques présents dans le Naḥw al-qulūb comparé avec les Lațāif va nous donner des indices de cohérence entre les deux ouvrages.

\section{GRAMMAIRE ET THÉOLOGIE.}

Certes, établir les liens qui ont poussé Qušayrī à s'intéresser à la grammaire jusqu'à développer une nouvelle application de la science de l'išăra, demeure difficile. T. Ivànyi souligne que l'effort pour établir un langage technique a fait naitre chez les soufis un

12. Risāla, p. 574.

13. V. Article « Ishāra » dans EI², vol. IV, p. 119. 
intérêt pour la grammaire ${ }^{14}$. On connaît bien la contribution de Qušayrī dans ce sens par sa Risāla. Il faut encore souligner que dans l'univers culturel de son époque les domaines du savoir étaient étroitement liés. La philologie s'affirmait de plus en plus comme instrument clé pour les autres sciences islamiques, qui, avec leur langage technique, donnaient des nouveaux outils linguistiques au développement de la grammaire. C'est notamment le cas $\mathrm{du}$ figh, dont les principes (ușūl) sont repris par les grammairiens ${ }^{15}$. Les appartenances théologiques étaient aussi source d'influence pour les théories grammaticales. Versteegh souligne que l'influence des mu'tazilites devient évidente quand on considère le rôle de la logique chez certains grammairiens comme al-Zajjājīi, al-Fārisī et Ibn Jinnī, auteurs qui ne cachaient pas leur appartenance à ce courant théologique ${ }^{16}$.

Dans les discussions intellectuelles de cette époque les disciplines sont liées et le Naḥw al-qulūb est un reflet de cette richesse d'échanges et d'oppositions. Un homme intellectuel comme Qušayrī, versé dans plusieurs disciplines et domaines du savoir islamiques a peutêtre ressenti la nécessité de montrer un possible lien entre grammaire et mystique. Le texte montre des thèses ašarites sur la nature de l'action humaine, sur les attributs divins - thèses rapprochées de celles de la pensée soufie de Qušayrī - qui essaie une synthèse entre les branches du savoir islamiques. La grammaire des cœurs est une proposition de solution soufie aux problèmes que la théologie avait reversé sur le langage. Dans cette nouvelle clé, Qušayrī indique sa propre solution, lorsqu'il intériorise les problématiques philosophiques du langage. Par exemple, la notion ašarite d'identité entre ism et musammā, dans le cadre des attributs divins, donne lieu à une réflexion sur la pratique de noms divins par le dhikr. La technicisation des terminologies propres aux sciences islamiques avait aussi éloigné ces sciences de leur fin originelle, la connaissance de Dieu. Le travail étymologique qu'on retrouve dans le Nahww al-qulūb vise à redonner aux mots toute leur portée, perdue dans la lourdeur de la technicisation linguistique (istișlāh). Cet effort de synthèse et d'apologie de la méthode soufie comme le seule garant de la revivification du vrai savoir spirituel est le trait fondamental de l'activité de Qušayrī, et on le retrouve aussi dans les pages du Naḥw al-qulūb.

Le Naḥw al-qulūb al-șagìr n'analyse pas toutes les règles de la grammaire arabe. Qušayrī a écrit un autre texte, plus exhaustif, le Naḥw al-qulūb al-kabīr, édité par Ibrāhīm Basyūnī et Aḥmad 'Alam al-Dīn al-Jundī au Caire, sur la base de plusieurs manuscrits ${ }^{17}$. On ne dispose pas d'éléments suffisants pour comprendre la relation entre les deux textes. De même,

14. IVÀNYI T., « Towards a grammar of the heart : al-Qušayrī’s Nahw al-qulūb », in Proceedings of the Colloquium on Logos, Ethos, Mythos in the Middle East \& North Africa, Part One, Budapest 18-22 September 1995, ed. K. Dévényi et T. Ivànyi, Budapest, 1996, p. 40-54.

15. «Ein andere wichtiger Einfluß war der des Rechts. Die Rolle des Grammatikers als eines « Rechtkundigen » (faqīh), der über die Korrektheit der Sprache sowie über die Korrektheit der grammatischen Beweise urteilt, wurde erweitert, und man versuchte nach dem Beispiel der Systematisierung im Recht (figh) ein konsistentes System der linguistischen Grundformen (ușūl) aufzubauen, wobei man auch in Einzelheiten die grammatischen Kriterien denen des figh nachbildete ». VeRsteEgh C.H.M. : « Die Arabische Sprachwissenschaft », in Grundriss der arabischen Philologie, I. Sprachwissenschaft (éd. W. Fischer), II. Literaturwissenschaft (éd. Helmut Gätje) et III. Supplement (éd. W. Fischer). Wiesbaden : L. Reichert, 1982, 1987 et 1992, V. 2, p. 161.

16. VeRSTEEGH C.H.M., ibid.

17. Ibrāhīm Basyūnī et Aḥmad 'Alam al-Dīn al-Jundī (éd. par), Naḥw al-qulūb al-kabīr, Le Caire, 1994. 
une datation de la composition des deux textes est impossible. Le Naḥw al-qulüb al-kabìr contient 60 sections contre 19 dans le Sạgìr. On voit que Qušayri y développe ses išărāt sur une échelle beaucoup plus vaste. Si le Naḥw al-qulüb al-șagìir se présente comme un recueil d'aphorismes sur la voie soufie, le Kabir montre une prose plus explicite, un souffle plus étendu. Qušayrī rentre dans les détails, son analyse des išarāt lui permet aussi de prendre de nouvelles directions interprétatives, par rapport au Șaghìr. Les deux textes montrent aussi une cohérence très forte au niveau de la méthode spirituelle. Le Kabir est un autre exemple du soufisme tel que le conçoit Qušayrī, et qu'il développe dans sa Risāla. Ici comme dans le Șagìir, les mutations linguistiques des mots sont le miroir des changements d'état de l'âme dans son parcours de purification.

\section{L'HÉRITAGE DU NAHW AL-QULŪB}

Quel est le rôle des interprétations allégoriquee du langage technique de la grammaire en tant que genre littéraire dans l'histoire du soufisme? Serait-il possible d'en tracer une histoire au sein de la littérature mystique? Cette question nous ouvre de nouveaux champs de recherche. Jusqu'à aujourd'hui, on connaît, à partir d'une époque plus tardive, une reprise de ce genre, surtout au Maghreb. M. Chodkiewicz signale que l'œuvre d'Ibn 'Arabī reflète un intérêt pour la portée symbolique de ce langage ${ }^{18}$. On peut signaler ici le Šarh al$\bar{A}$ ğurrumiya du soufi marocain Ahmad al-Zarrūq $\left(X^{\text {ème }} \text { siècle }\right)^{19}$, un deuxième commentaire soufi toujours de la Āğurrūmiya composé au XIX ${ }^{\text {ème }}$ siècle par Aḥmad b. 'Ağība ${ }^{20}$. Le texte d'al-Āğurrūm a été aussi objet d'un commentaire soufi par 'Alī b. Maymūn al- Fāsī (853917/1450-1511)21. L'intérêt des soufis pour ce texte doit être remarqué. Ici on se limitera à signaler que al-Širbīnī, dans son šarh grammatical, dit bien que Āğurrūm signifie șūfĭ, faqīir. ${ }^{22}$ On retrouve la mention de certaines notions relatives à la différence entre le iṣlāḥ al-lisān et

18. «La terminologie des grammairiens, sous la sécheresse de son apparence, est riche d'un symbolisme dont Ibn 'Arabī utilise toutes les ressource. Ainsi en va-t-il de la banale distinction entre consonnes et voyelles. Ces derniers, comme l'indique leur nom (harakat) ont pour rôle de «mouvoir » le consonnes inertes; elles leur donnent vie de même que l'insufflation de l'Esprit divin anime la forme adamique tirée de l'argile (Cor. 15 :29). Mais la manifestation, orale ou écrite, de la consonne considérée est affectée par cette vocalisation ; sa réalité essentielle (haqiqa) est immuable. La relation entre, par exemple, le dal final de Zayd et les voyelles brèves qui en déterminent la fonction dans le discours est par conséquence analogue à celle qui existe entre nos propres essences - nos a'yan thabita - et les formes successives qui les manifestent ad extra [...]» . Michel CHoDKIEwIcz, Les illuminations de la Mecque, textes choisis des Futūhāt Makkiya (avec la collaboration de W. Chittick, C. Chodkiewicz, D. Gril et J. Morris), Paris, Sindbad, 1988, p. 51-52. Voir aussi, dans le même volume, l'introduction de D. GRIL à sa traduction du chapitre des Futūhāt sur la science des lettres, p. 198-228.

19. Signalé par Ali LAHмI KнUSHAIM dans son Zarrūq the șüfi. A biographical and critical study of a mystic from North Africa, Tripoli, 1976.

20. Aḥmad в. 'AĞīBa, Tağrīd šarh matn al-Āğurrūmiyya, Al-mațba'a bi-Mișr, 1419 (h). Des extraits ont été traduits par J. L. Michon, Le soufi marocain Aḥmad ibn 'Ağïba (1746-1809) et son Mírāj, Paris, II. éd., 1990.

21. Al-Ghazlanı, A. : « Présentation et édition critique de la Risālat al-maymūniyya fì tawhīd al-ājurrūmiyya de 'Alī b. Maymūn al-Fāsī (853-917/1450-1511) », mémoire de maîtrise, Université de Provence Aix-Marseille I, 1997-1998.

22. «[...] Ibn Āğurrūm (spelt with ' followed by $\bar{a}$ and double $r$, which is a Berber expression meaning faqīr or șūfí) [...]». Trad. de M. G. Carter, Arab Linguistics. An introductory classical text with translation and notes, Amsterdam, 1981, p. 4. 
le ișlăh al-qulūb dans l'oeuvre du maître soufi algérien Ahmad b. 'Aliwa, au début du XX ${ }^{\text {ème }}$ siècle $^{23}$. On sait donc que le Naḥw al-qulūb a laissé un héritage. La question à laquelle il faudrait pouvoir répondre est de savoir s'il a eu des antécédents. D'ultérieures recherches sur ce genre littéraire pourraient nous renseigner sur l'originalité de cet ouvrage.

\section{E. LES ÉDITIONS DU TEXTE}

On dispose de deux éditions du texte du Naḥw al-qulūb al-șagìr : l'une est due à Ahmed Alam Eddine Goundi, éditeur aussi du Nahw al-qulüb al-kabir, paru à la Maison Arabe du Livre, en 1977, à Tunis ; l'autre, plus récente, date de 1996 et a été établie par Tamàs Ivànyi et a paru parmi les actes du colloque «Logos, Ethos, Mythos in the Midle East \& North Africa » qui a eu lieu a Budapest en 1995. Cette dernière édition apparemment n'a pas de lien avec l'édition tunisienne, mais son auteur, Tamàs Ivànyi, dans une note dit qu'après la rédaction de son article, il a pris connaissance de l'existence de l'édition tunisienne du Nahww al-qulūb al-șagìr par l'édition du Naḥw al-qulūb al-kabīr édité par Ibrahim Basyūnī et Ahmed Alam Eddine Goundi. Notre traduction suit l'édition de Tunis.

23. Ahmad al-'Alawī, Knowledge of God: A Commentary on al-Murshid al-Mu'in of Ibn al-Ashir, Abd as-Sabur al-Ustadh (editor); Abd al-Kabir al-Munawarra, Abd as-Sabur al-Ustadh (translators); Shaykh Abdalqadir as-Sufi al-Darqawi (introduction), Madinah Press , 2005. Voir l'introduction de l'auteur. 
TRADUCTION DU NAHW AL-QULŪB

\section{INTRODUCTION}

La louange est à Dieu, qui a confié la sagesse à ceux qui en sont dignes, qui a enseigné à Adam tous les noms ${ }^{24}$ et lui a fait connaitre le sens du cercle de l'existence, si bien qu'il en a résolu la difficulté. Il a alors explicité à ses fils les lettres [du cercle], la marque de leur nom (ism) et la trace de leur acte (fil). Il en est qui ont redoublé d'efforts pour obtenir une part telle une pluie abondante sans se contenter d'une légère ondée ${ }^{25}$. D'autres ont accepté de nouer une résolution ferme ('azìma) mais s'y étant engagé, l'ont défaite. Un groupe, pour manifester son excellence, s'est orienté vers la correction de la langue. Un autre, s'est dirigé au-delà vers les jardins de l'âme, où poussent les branches de la désobéissance sur l'arbre de l'excès. Il l'a coupé à la base, puis a suivi la voie (nahw) de celui qui a montré la faiblesse de l'âme afin d'en obtenir la guérison et de s'adresser directement à elle ${ }^{26}$. Je Le loue pour tous Ses grâces que Sa générosité a dirigées vers moi et elles m'ont fait don de leur pluie fécondante. Je témoigne qu'il n'est de dieu si ce n'est Dieu, seul sans associé et je m'abriterai à l'ombre de ce témoignage le jour où il n'y aura d'ombre que la Sienne et je témoigne que notre seigneur Muhammad est Son serviteur et Son envoyé. Il l'a envoyé pour défaire les armées des faux dieux et pour avilir les lions de l'idolâtrie. Que Dieu lui accorde ainsi qu'à sa famille et ses compagnons une grâce sans fin jusqu'au jour où tout être remettra ce qui il porte en lui ${ }^{27}$.

Le terme nahw indique le but, la direction. Or les hommes se différencient par leurs orientations et sont différents selon les aiguades où ils se rendent et dont ils reviennent. Pour l'un, la correction de la langue (taqwìm lisānihi) représente le terme de la science. Pour un autres, la correction du cœur (taqwìm janānihi) représente la totalité de son occupation et de son effort. Les premiers sont ceux qui suivent l'expression explicite (șāhib al-'ibāra) ; les seconds sont ceux qui suivent l'allusion (șāhiib al-išāra).

\section{LES PARTIES DU DISCOURS (aqsām al-kalām).}

Les gens du sens obvie disent: les parties du discours sont au nombre de trois : nom, verbe et particule. Les gens du sens allusif disent : les fondements (ușūl) [de la voie] sont au nombre de trois : paroles, actes et états. Les paroles sont les sciences qui précédent l'action, car le Prophète a dit : « On m'a ordonné de combattre les gens jusqu'à ce qu'ils disent : « Il n'y a pas de dieu si ce n'est Dieu. S'ils le disent, ils mettent à l'abri de moi leurs vies et leurs

24. Cf. Coran 2, $31:$ «Et Il enseigna à Adam tous les noms. »

25. Cf. Coran 2, 265: «Ceux qui dépensent leur biens par souci de plaire à Dieu et pour affermir leur âmes sont comparables à un jardin situé sur une colline, sur lequel s'est déversé une pluie abondante et qui a donné un double de fruits. Si le jardin n'est pas arrosé par la pluie, il l'est par une rosée, et Dieu est Clairvoyant au sujet de ce que vous faites. »

26. Ce passage n'est pas clair : wa-yuhātibuhā šifāhan wa-man lahā, autre variante : tuhātịibuhā.

27. Cf. Coran 22, 2 : « Le jour où toute femme enceinte accouchera ce qu'elle porte en elle». 
biens, sauf pour ce que le droit exige d'eux ». Il faut ensuite s'empresser d'accomplir les œuvres pieuses, viendront alors les états spirituels, dons de Dieu ${ }^{28}$.

\section{LES NOMS ET LEUR DÉRIVATION (al-asmā' wa-ištiqāquhā).}

Les gens du sens propre disent: le nom (ism) est dérivé de l'élévation (al-sumuww) ou de la marque (al-sima), selon la divergence à ce sujet. Les gens du sens allusif disent : le nom du serviteur est ce par quoi Dieu l'a marqué dans la prééternité de Sa Volonté : malheur ou félicité. La valeur de celui que Dieu, parmi les créatures, a rapproché dans la prééternité de Sa Volonté est élevée ${ }^{29}$. Lorsque les serviteurs entrèrent à l'école de l'enseignement divin, Adam prit connaissance de la Tablette de l'Existence et lut : «Et Il enseigna à Adam tous les noms » (Coran 2, 22); Muhammad - sur lui la grâce et paix - prit connaissance de la Tablette de la Vision Contemplative et il lui fut dit dans le langage de l'état (bi-lisān al-ḥāl) : « Nous te faisons connaître tout être existant». Puis il reçut cette parole : « Récite au Nom de ton Seigneur qui a créé » (Coran 96,1). Quand il eut récité, fut éduqué et amendé, il lui fut dit: «O Muhammad, tu Nous as connu par les Noms et les Attributs, apprends à Nous

28. Ce passage suit la division traditionnelle du discours. Le texte du Naḥw al-qulūb n'approfondit pas la question des catégories du discours. Le style concis qui caractérise cet ouvrage réside dans la volonté de Qušayrī de ne pas s'attarder sur l'isțilăh, le lexique technique de la grammaire. Il essaie au contraire de revenir au sens premier du mot, et à partir de là, de dégager une signification spirituelle. Ce bref paragraphe cache une forte parenté doctrinale avec l'ensemble du système de pensée de Qušayrī. Cette perspective est le reflet de la complémentarité entre hāal et maqām. Si on cherche dans la Risāla une définition de ces deux concepts clé du soufisme, on retrouve la notion de hâal liée à celui de mawāhib:

"L'état [spirituel] chez les soufis (al-qawm) est quelque chose qui parvient au cœur, sans qu'ils en fassent en effort. Ils ne peuvent ni l'attirer, ni l'acquérir. [...] Les états (ahwāl sont des dons (mawāhib), alors que les stations (maqāmāt) sont des acquisitions. Les états arrivent directement du Généreux ; on parvient aux stations (maqūmāt) par la dépense de l'effort. » (Risāla, p. 92.)

L'exhortation à l'accomplissement des œuvres rentre dans le cadre de la théologie de Qušayrī, selon laquelle la foi correspond aux actes. Dans le Luma' fil l-ítiqād, bref credo ash'arite composée par Qušayrī, on retrouve cette définition classique :

«La foi, c'est la reconnaissance par l'intellect, l'action par le corps et l'affirmation par la langue. » (R. M. Frank, « Tho shorts dogmatics works of Abū l-Qāsim al-Qušayrī : Al-luma' fì l-ítiqād », MIDEO, tome 15, 1982, p. 70.)

29. Qušayrī propose deux interprétations traditionnelles de cette étymologie, l'une de l'école de Basra, l'autre de l'école de Kūfa. Pour la première, le terme ism dérive de al-sumuww (SMW, «élévation »). Pour l'école de Kūfa, ism est un dérivé de sima (WSM wasm, signe, marque, qualité, caractéristique). Qušayrī passe, dans son išāra, au lien entre le nom et le nommé. Le nom est en effet celui du serviteur (ism al-'abd). Le nom fait allusion à la « caractérisation » de son destin de toute éternité. Il est la nature même du serviteur, homo nomen. Il s'agit de la nature intime de l'être, en relation avec la 'ubūdiyya. Le passage du Nahww al-qulūb, même si fortement imprégné d'implications théologiques et doctrinales, dans sa brièveté, reste un aphorisme sur la façon du soufisme de traiter des questions d'ordre théologique. Il nous renvoie à de longs débats avec le mu'tazilisme (voir article « kadar » dans l'EI²), mais il les dépasse aussitôt, pour viser l'essentiel. On retrouve la discussion sur l'étymologie du terme « ism » dans le Al-tahsīn fì al-tadhkīr (éd. par Muhammad b. 'Abd al-Hādī al-Farūqī, Dār al-Bayrūtī, Damas, 2003, p. 29), et également dans les Lațāi if al-išārāt. Dans le commentaire à la sourate 13, il écrit :

«Le nom (ism) vient de « marquer » (wasama). Celui qui marque son être extérieur de la servitude et sa conscience intime de la contemplation de la Seigneurie, son aspiration s'élève vers les hautes stations et son degré est rapproché des demeures sublimes. En effet ism est dérivé de la marque (sima) ou de l'élévation (al-sumuww ).» (Lațāifif, vol. 2, p. 190.) 
connaître par l'Essence de " Récite et ton Seigneur et plus généreux» (Coran 96, 3) », « Dis : Allah! Puis laisse-les s'amuser à leur bavardage » (Coran 6, 91) ${ }^{30}$. Absent au Nom, il trouva le Nommé. Se détournant de l'acte, il dénoua l'énigme de la lettre, c'est à dire le sens qui ne peut être nommé ${ }^{31}$.

LE NOM : «SAIN » OU « DÉFICIENT » (șahịh wa-mu'tall).

Le nom est sain (șahịh ) ou déficient (mu'tall ) : Les gens du sens propre disent : sain est le nom qui n'est pas affecté par l'une des lettres de la déficience : le alif, le $w \bar{a} w$, le $y \bar{a}$. Pour les gens du sens allusif, c'est celui dont le nom n'est affecté ni de l'alif de l'ambiguïté (ilbās), ni du wāw de la suggestion psychique (waswās), ou du y $\bar{a}$ ' du désespoir (yā's) ${ }^{32}$. Le nom alors est sain et peut recevoir la flexion casuelle (írāb). Ceci signifie le sens clair (al-bayān), puis le dévoilement (kašf) et la vision directe ('iyān). Il a d'abord la science de la certitude ('ilm al-yaqin), puis l'œil de la certitude ('ayn al-yaqin) et enfin la vérité de la certitude (haqq alyaqin). Mais Dieu est plus savant ${ }^{33}$.

30. La deuxième partie de ce paragraphe passe de l'étymologie grammaticale du nom, à l'origine des noms sur un plan métaphysique. On retrouve deux sortes de noms : ceux révélés à Adam et la science de Muhammad, qui tire son origine de la Tablette de la Vision Contemplative (lawh al-šuhūd), qui renvoie à la connaissance du divin, tandis que la Tablette de l'Existence (lawh al-mawğūd) représente la connaissance de la création. Le passage indique une progression dans le processus de connaissance. Qušayrī montre que le parcours de la connaissance procède par étapes et degrés : on passe de la récitation à une transformation, selon la progression de la voie. Le dernier verset cité renvoie au dikr : Qušayrī relie un discours sur les attributs divins à ce qui l'intéresse le plus : l'expérience de la connaissance.

31. La station ultime voit la disparition du Nom pour laisser apparaître le Nommé, Dieu. Dépasser l'acte permet de " dénouer l'énigme de la lettre », l'accès au sens caché derrière l'aspect " extérieur » des noms. Cette dernière phrase est comme un retour circulaire au début du paragraphe, lorsque Qušayrī commence par cette distinction entre les deux réalités du nom. Le passage du Nom au Nommé est aussi possible parce que dans sa vision, la multiplicité des noms ne porte pas atteinte à l'unité divine. Dans son Šarh al-asmā', Qušayrī explique que par « nom » il faut entendre la dénomination (tasmiya). Pour l'aš'arisme, le nom (ism) est l'être nommé (al-musammā). Cette position est basée, dans la courrant aš̌arīte, sur l'interprétation des attributs de l'essence (șifät al-d̄att), qui, selon un point de vue opposé à celui des mu'tazilites, participent de la réalité de Dieu (Voir article « Mu'tazila » dans EI², VII p. 785-95). T. Ivànyi dans sa présentation du Naḥw al-qulüb, voit dans la relation entre nom et nommé une allusion aux niveaux de la 'ibāra et de la išāra:

« Le grammairien s'occupe des noms, le mystique de l'essence, qui est l'objet réel des noms. [...] La science de l'išāra nous ramène vers les sens cachés qui n'ont pas d'équivalent linguistique (lā yusammāa). » (Tamàs Ivànyi, op. cit., p. 43.)

32. La méthode utilisée par Qušayrī pour établir ce rapport symbolique, dans ce paragraphe se base sur les sens inclus dans les lettres de l'alphabet. La loi qui gère ce mécanisme symbolisant rend une simple lettre capable de représenter un concept entier, par analogie phonique. Cette méthode est aussi propre à certains commentaires des Lațāifi à propos des lettres isolées.

33. Qušayrī insiste sur le fait que ces défauts empêchent la vraie manifestation du írāb. Dans ce paragraphe, l'írāb se fait l'image d'un processus de connaissance (grâce à sa relation sémantique avec le terme bayān). Les trois défauts de l'âme sont remplacés par les trois degrés de la connaissance. La progression bayān, kašf, 'iyān est mise en relation avec la série 'ilm al-yaqin, 'ayn al-yaqìn, haqq al-yaqīn. Les degrés de la certitude (yaqīn) sont analysés dans un paragraphe spécifique de la Risāla, qui décrie une relation similaire :

«Le 'ilm al-yaqin est certitude, ainsi que le 'ayn al-yaqin et le haqq al-yaqin : ils sont la même certitude (nafs al-yaqin). Le 'ilm al-yaqīn, dans leur terminologie technique (iștilāhihim), est ce qui est fondé sur un argument (burhān). Le 'ayn al-yaqīn est ce qui est démontré avec évidence (al-bayān). Le haqq alyaqīn est ce qui est accessible par la vision [directe] (al-'iyān).» (Risāla, p.171.) 
LES CAUSES EMPÊCHANT LA FLEXION NOMINALE (mawāni' al-șarf).

Les causes qui empêchent la flexion nominale (írāb), pour les gens du sens propre, sont au nombre de neuf et sont bien connues ${ }^{34}$. Chez les gens du sens allusif, ce sont:

- Le pluriel (al-jam', lit. « la réunion ») : le savant doit s'abstenir de réunir [les biens] de ce monde et se garder de la réunion des hommes autour de lui.

- Al-șarf (lit. « détourner, changer») : attirer les regard sur soi.

- Certaines formes de l'adjectif qualificatif (al-wașf) : le désir d'être qualifié et connu par le bien.

- Le nom propre féminin (al-ta'niț $)$ : la faiblesse de la résolution et l'acceptation des choses viles.

- La détermination (al-márifa, lit : la connaissance) : connaître la grâce de Dieu mais manquer de gratitude.

- L'origine non arabe (al-ujma) : négliger les bienfaits divins en cachant sa science.

- Le « déviation » d'une forme vers une autre (al-'adl) : son détournement de la voie droite.

- Le nom composé (al-tarkīb) : altérer sa science par des actes d'ignorance.

- La lettre alif: l' alif du « moi » (anā).

- La lettre nūn: le nūn des noms de majesté.

- Certains schèmes verbaux (wazn al-fil) : peser (yazin) ses œuvres en pensant avoir obtenu quelque chose, et éprouver de l'autosatisfaction ('ujb).

Lorsque que deux de ces défauts se réunissent, il ne se «tourne» pas [lit. «il ne se fléchit pas - lam yanșarif»] vers l'agrément divin (qabül) et se détourne de la porte de l'union.

\section{LA FLEXION NOMINALE ET L'INVARIABILITÉ (al-i'rāb wa-l-binā')}

La flexion nominale (al-i'rāb) s'effectue à partir de trois voyelles (lit. « mouvements », harakāt) : l'élévation ( $r a f$ '), l'établissement horizontal (naṣb), l'attirance vers le bas (jarr) et l'apocope (jazm). Les gens du sens allusif élèvent leurs aspirations vers Dieu, dressent leurs corps (naṣb abdānihim) dans l'obéissance à Dieu, baissent (hafạ) leurs âmes pour s'humilier devant Dieu, coupent leurs cœurs de tout ce qui est autre que Dieu et leur quiétude (sukūn) est en Dieu. Le décliné (al-mu'rab), c'est celui [dont le cœur est] soumis au changement

34. Il s'agit de noms diptotes qui ne suivent pas la déclinaison à trois désinences. Le nombre des causes empêchant la flexion nominale peut varier selon les auteurs ; le Al-Naḥw al- wāḍh (ed. par 'Alī al-Ğarim et Muștafā Amīn, Dar al-ma'ārif, vol. 3, p. 126) en mentionne six : le nom propre féminin, le nom d'origine non arabe ( $a^{\prime}$ ğamì), le nom composé (murakkab), le nom qui termine par alif et nūn (ex. 'Uthmān), le nom qui ressemble à une forme verbale (ex. Ahmad), les noms qui ne suit pas une dérivation normale comme le schème " fu'al » (ex. 'Umar). Pour des sources exhaustives concernant cette question voir Sibawayh, al-Kitāb, Beyrouth, 1967, p. 4-70; al-Zağğāğ, Mā yanșarif wa mā lā yanșarif, éd. H. Qarā‘a, Le Caire, 1971; al-Zağǧāăḡi, al-Ğumal. Précis de grammaire arabe publié avec une introduction et un index par Mohammed ben Cheneb, Paris 1957, p. 224-234. 
parmi les gens de la « coloration » (talwīn); l'invariable (al-mabnī), c'est celui dont l'état est stable et invariable et ainsi sont ceux qui ont atteint l'établissement ferme (tamkīn) ${ }^{35}$.

DÉTERMINATION ET INDÉTERMINATION (al-asmā̄' : ma'āarif wa-nakirāat) ${ }^{36}$.

Les noms sont déterminés ou indéterminés. De même, parmi les adorateurs il y a celui qui est connu (márūf), qui a une place parmi les Initiés, est connu pour cela et qui a atteint une station de véridicité (maqām al-ṣidq) par laquelle il est qualifié. Il en est qui ne sont pas connu, qui n'ont pas de part avec les Initiés et ne recherchent rien d'autre que la nourriture et le sommeil ${ }^{37}$.

\section{LE SUJET DE LA PHRASE NOMINALE (al-mubtada').}

Le sujet de la phrase nominale (al-mubtada') est «élevé » (marfū') car il est dépouillé des régents grammaticaux ( al-'awāmil al-lafziya). Le pauvre, dépouillé de tout, est à un rang

35. Pour l'établissement des išārāt tirées des images relatives à la flexion nominale, Qušayrī exploite le champ sémantique déjà présent dans le vocabulaire technique de la grammaire : raf ', naṣb et ğarr / hafḍ. Cette terminologie se prête à l'allusion aux traits essentiels de l'engagement dans la voie spirituelle: élévation des aspirations, établissement des corps, abaissement des âmes devant Dieu. Les mouvements extérieurs dans la grammaire du langage sont assimilés aux mouvements intérieurs de la grammaire des cœurs. La flexion nominale (irāb), dans un paragraphe précédent était signe de la "pleine » manifestation de la connaissance, ici à l'írāb symbolique est attaché un trait d'imperfection. Le couple antithétique mu'rab - mabnī suggère à l'auteur une comparaison avec les états de tamkin et talwīn. Dans la Risāla, on trouve un chapitre consacré à ces deux termes :

«La coloration (changement, talwīn) est la qualité propre de ceux qui ont des états mystiques. Létablissement ferme (tamkin) est la qualité de ceux qui ont réalisé les vérités supérieures. Toute la durée de son cheminement, le serviteur est soumis au talwìn, parce qu'il monte d'un état à un autre et passe d'une qualification (sifa) à une autre. Il sort d'une station pour arriver à une halte. Lorsqu'il est parvenu, il s'établit d'une façon permanente. [...] Celui soumis au talwīn se trouve toujours dans la croissance (ziyāda), les gens du tamkin sont parvenus, et sont dans l'union. Le signe qu'ils sont parvenus est l'absence totale de préoccupation. Un maître a dit : le voyage de ceux qui cherchent est terminé lorsqu'ils ont vaincu leur âmes, car par cette victoire ils parviennent au but. Le maître a dit : il veut dire que les statuts de l'humanité se retirent et le pouvoir de la réalité supérieure s'impose (istīlä' sultān al-haqìqa). Si le serviteur reste stable dans cet état, il obtient le tamkīn. [...] Sache que le changement (taǵayyur) atteint le serviteur pour deux raisons : à cause de la force de l'évènement spirituel (quwwa al-wārid), ou à cause de sa propre faiblesse. La quiétude (al-sukūn) peut avoir aussi deux raisons : sa propre force intrinsèque ou la faiblesse de l'évènement spirituel. » (Risāla, p. 162163.)

La thématique du talwīn/tamkīn est toujours présente dans les Lațā’if. Dans son commentaire du verset 23 de la sourate Le Fer, l'auteur explique que la propension des hommes à changer d'état face à ce qui leur arrive (wārid), est un critère sur lequel on peut qualifier les différents états spirituels des hommes :

«Celui qui ne change pas face à ce qui lui arrive (wärid), en bien comme en mal, comme épreuve ou éloignement est parfait [...]. Il est maître de son instant (sayyid waqtihi). [...] Le changement (tagayyur) est le signe que l'âme subsiste (baqā' al-nafs). » (Lațāif, vol. 3, p. 292.)

36. Lit. «connu» et «inconnu».

37. Ma'rūf (connu et déterminé) signifie aussi « reconnu comme bien », munkar (inconnu et indéterminé, nākir z a) signifie aussi « objet de réprobation». La notion centrale est celle du maqām al-șidq, la station de la véridicité. C'est par cette station que le serviteur affilié aux initiés est reconnu et qualifié. Cette notion clé du tasawwuf se retrouve dans divers passages de la Risāla et des Lațā'if. Le maître de Qušayri, Abū 'Alī al-Daqqāq, définit la sincérité ainsi : «Que tu sois avec les gens ainsi que tu te vois toi-même, ou que tu te vois tel que tu es (an tarā min nafsika kamā takūn). » (Risāla, p. 345.) 
élevé (marfū' al-qadr) et de même « ce qu'on rapporte à son sujet » (habaruhu), car il a coupé les liens et s'est attaché aux réalités supérieurs (haqā’iq) qui procèdent du Créateur ${ }^{38}$.

\section{LES TEMPS VERBAUX.}

Il y a trois sortes de verbes : passé (mādī), présent (hāl), futur (mustaqbal). Ainsi les états des Initiés (al-qawm) sont différents. Parmi eux, certains engagent leur méditation (fikra) sur ce qui précède leur vie (sābiqa), les autres sur ce qui la conclut (hātima) et d'autres enfin s'emploient à reformer l'instant où ils se trouvent, par delà la réflexion sur le futur et le passé ${ }^{39}$.

\section{L'INDICATIF PRÉSENT (al-ḥāl al-marfū').}

Le verbe au présent (al-ḥāl) est « élevé » (marfū') s'il n'est pas précédé par une particule du subjonctif (nāṣib) ou de l'apocopé (jāzim) ${ }^{40}$. Ce qui l'« établit horizontalement » (al-nāṣib) est la vision que le serviteur a de son acte. Ce qui le coupe (al-jāzim) est l'interruption de son cheminement spirituel. Lorsque le serviteur est à l'abri de la prise en considération de ses œuvres et de l'interruption [du chemin], sa valeur s'élève auprès du Tout-Puissant, Très-Pardonnant. « Vers Lui monte la bonne parole et l'œuvre pieuse, Il l'élève. » (Coran 35, 10) ${ }^{41}$.

38. La définition donnée par Qušayrī du mubtada' est négative : tout ce qui n'est pas géré par un régent grammatical. Cette terminologie est à la base de la išăra de Qušayrī, qui se fonde sur le sens propre de tajrīd, " privation de », « dépouillement». Le sens propre est néanmoins repris dans son utilisation propre par le soufisme, qui a fait du tajrìd le « dépouillement» intérieur pour atteindre la station du faqr. Cet abaissement du faqìr est l'équivalent inversé d'une élévation de rang auprès de Dieu. On voit qu'ici Qušayrī ne cherche pas une allusion spirituelle dans le terme mubtada', le « début ». Il se concentre sur sa réalisation grammaticale en tant que marfūं. Pour donner un exemple de développement différent, on peut citer ici le Šarh al- Ājurrūmiyya du soufi marocaine Aḥmad b. 'Ağība (m. 1809), qui a une orientation différente du Nạ̣w al-qulūb. Comme on l'a déjà signalé, les išārāt de Qušayrī, dans cet ouvrage, sont en relation avec les états du murīd et ses degrés de proximité ou d'éloignement. Dans le šarh d'Ibn 'Ağība, les allusions spirituelles sont, comme expliqué par J. L. Michon, « des symboles cosmogoniques et théurgiques jouant un rôle précis dans le processus de la manifestation divine. Ainsi, la langue toute entière apparaît comme une théophanie, un ensemble cohérent de significations spirituelles où la lumière du Maître des mondes s'irradie pour l'édification et la guidance des créatures. » (J. L. Michon, op. cit., p. 115.)

39. Les temps verbaux sont pris ici comme allusion aux ahwāl, les états dans lesquels se trouve l'homme. Le waqt c'est le premier des termes technique du soufisme (ișțilāhat) cités dans la Risāla. Ce processus de centralisation dans l'instant est résumé dans la parole "al-șūfí ibn al-waqt », cité par Qušayrī (Risāla, p. 130) Une autre parole de son maître Abū 'Alī al-Daqqāq rappelle le passage du Naḥw al-qulūb: « Le temps, c'est ce dans quoi tu te trouves (al-waqt: $m \bar{a}$ anta fihi). Si tu te trouves dans l'ici-bas, ton temps est l'ici-bas; si tu es dans l'au-delà, ton temps est l'au-delà. » (ibid.) Il rapporte encore cette définition du waqt: «L'instant (waqt) est ce qui est entre deux temps (zamanayn) : le passé et le futur. » (ibid). L'engagement à une réflexion sur l'instant présent est contenu aussi dans cette phrase : "Le pauvre (al-faqir) ne s'occupe pas de l'instant passé ou de celui à venir, quant l'instant dans lequel il se trouve » (ibid). Cet ensemble de citations replace le passage du Naḥw al-qulūb dans l'univers doctrinal d'Qušayrī et dans son école de soufisme. La Risāla fait aussi un lien entre waqt et aḥwāl, comme suggéré par la réflexion du Naḥw al-qulūb sur les temps verbaux. La maîtrise de son waqt correspond au passage de la phase du talwìn à celle du tamkin, comme on l'a déjà vu précédemment.

40. Le nāṣib, « ce qui met au cas direct » pour les noms et au subjectif pour les verbes signifie littéralement : ce qui établit. Le ğāzim, « ce qui met à l'apocopé » signifie littéralement « ce qui coupe ».

41. Cette section du Naḥw al-qulūb a comme départ l'ambivalence sémantique du mot « ḥăl», qui renvoie à l'état spirituel et, dans la terminologie grammaticale, au « présent ». Dans le paragraphe précèdent Qušayrī met l'accent 
L'AGENT (AL-FĀ'IL) ET LE COMPLÉMENT (al-maf'ūl ).

L'agent ( $\left(\bar{a}^{\prime} i l\right)$ est élevé (marfü') [au cas nominatif] et le complément (maf'ūl) est « établi » (manșūb) [au cas direct]. Lorsque que le connaissant voit qu'il n'y a pas d'autre agent que Dieu, sa valeur grandit ${ }^{42}$ et sa mention est élevée ${ }^{43}$. Il est sous l'emprise de sa Majesté, il s'humilie lors de la vision de Sa Perfection, il voit son âme objet [de l'action divine] (maf ūlan) et se dresse pour L'adorer : «Lorsque tu as fini, continue à œuvrer et pour ton Seigneur sois plein de désir. » (Coran 90, 7-8) ${ }^{44}$.

\section{LE COMPLÉMENT CIRCONSTANCIEL D'ÉTAT (al-ḥāl).}

Le complément circonstanciel est une qualification de ce qui a l'aspect du sujet et de l'objet (wașf hay'a al-fā'il wa-l-maf'ül). La condition est d'être indéfini et au cas direct (nakira manșūba). Le connaissant (al-'ārif) a orienté son être vers Dieu pour l'amélioration de son état, grâce à des efforts qui visent à faire méconnaitre son âme (fi tankìr nafsihi), afin de ne pas être connu. Ses états avec Dieu sont droits et solidement établis (mustaqìma

sur la notion d'acte au présent, le waqt qui qualifie le soufi d'une adhérence pleine à son instant présent. Ici il développe encore le thème de l'instant présent, mais sous autre point de vue. Le marfü est décrit en négatif : tout ce qui n'est pas introduit par une particule qui altère le degré d'élévation. L'altération se produit sur deux niveaux: un passage du plan "vertical », d'une adoration pleine - dont «l'état élevé » (hāl marfū') est l'image - à un plan « horizontal » (nașb), la vision par le serviteur de ses propres actes. Le troisième degré est la rupture (ğazm) de la voie. Cette rupture empêche la manifestation de la conjugaison verbale. Comme dans le cas de l'írāb, la manifestation graphique et phonétique, selon les trois niveaux- est la marque de la voie, et le sukūn-pour le verbe le jazm - de son arrêt. Le discours sur la vision du serviteur sur ses propres actions est reprise dans la Risāla, dans la section consacrée au jam' et au farq. Le farq relève de l'agir humain - accomplissement de la 'ubüdiya de ce qui convient aux états de la nature humaine. Qušayrī parle de plusieurs degrés de jam', notion qui donc prévoit une échelle de réalisation. Létat le plus bas dans cette réalisation est représenté par la vision des actions. Le fait que Dieu montre au serviteur ses actes d'adoration est un signe de séparation («'abd bi-wașf al-tafriqa », Risāla, p.143). D’une façon complémentaire, l'absence de la vision de ses propres actes est signe de intimité (uns) avec Dieu : comme dans ce passage du Nahww al-qulūb, le jam', station plus élevée que la précédente, correspond à un changement de vision. Celui qui voit l'agir, même le propre, comme une action divine, celui auquel Dieu montre son propre agir, porte la marque de l'union. L'absence de vision des actes correspond à la station décrite par Ibn 'Ağỉba comme fanā' fi-l-af'āl : «état où l'on ne voit pas d'autre agent que Dieu » (J.L. Michon, op. cit., p. 233). On notera enfin que pour la première fois Qušayrī cite un verset coranique. Le verset du Coran fonctionne comme centre sémantique autour duquel l'auteur développe son išăra. Cette méthode était jusqu'à ce paragraphe absente. Le versetsouligne que l'élévation est une action de Dieu. L'œuvre pieuse ne s'élève pas par soi-même, mais par la grâce de Dieu : « et l'œuvre pieuse, Il l'élève ».

42. Al-Kalābād̄ī rapporte la définition suivante de la notion du qurb :

« Un soufi, interrogé à propos de la proximité, répondit : «Elle consiste dans le fait que tu Le vois agir en toi». Ceci signifie que tu regards Ses œuvres et Ses grâces sur toi, et en elles disparaît ta vision de tes actes et de tes efforts. " (Táarruf li-madhab ahl al-tașawwuf, éd. par A. J. Arberry, Maktaba alKhānğì, Le Caire, 1933, p.125.)

43. Allusion à Coran $94,4:$ « Nous avons élevée ta mention ».

44. Ce paragraphe développe la question des actes. Il y a une progression entre ce passage et le précédent. Si l'élévation indique la valeur du serviteur dans l'absence de vision de soi-même, ici le marfü est Dieu, en tant qu'agent universel. Les âmes sont soumises à son action et le serviteur doit en avoir conscience. Si auparavant le hạal marfü était une marque positive, opposé au manșūb de la vision de ses propres actes, ici le symbolisme est renversé. Le manșūb, dans cette nouvelle perspective, est un trait connoté positivement. Le symbolisme est ambivalent, il fonctionne dans les deux directions. Dans le développement du Nahw al-qulūb, une image récurrente est celle du double mouvement de l'abaissement de l'individualité (tawādu') suivie de l'élévation auprès de Dieu. Cette idée, est déjà présente dans un hadīth recueilli par Muslim : «[...]wa-mā tawāda'a ahadun li-llāh illā rafa'ahu Allāhu 'azza wağall ». D’après al-Nawāwī, Riyāẹ al-ṣāliḥin, bāb al-tawāḍu wa hafḍ̂ al-ğanāḥ li-l-mu'min. 
muntașiba $)^{45}$, cachés par le voile de la dissimilation. L'indétermination (al-nakira) est un voile : «L'ignorant les croit riche à cause de leur retenue (ta'affuf).» (Coran 2, 273) ${ }^{46}$.

\section{LA SPÉCIFICATION (al-tamyīz).}

La spécification est une explication de ce qui n'est pas clairement exprimé, un éclaircissement de ce qui n'est pas compris. Les Initiés (al-qawm) distinguent par la science le vrai du faux. Par le cheminement spirituel (al-sulük) ils distinguent celui qui porte la parure de la connaissance (al-ḥâlì) et celui qui en est dépourvu (al-'ạțil) La spécification vient lorsque le discours est achevé. De la même manière [les hommes] apprennent d'abord la religion (tafaqqahū), après s'isolent, maîtrisent la science, puis il se distinguent (tamayyazū). Lorsque le degré de la distinction (rutba al-tamyiz) atteint en eux la perfection, Dieu les « établit " ${ }^{47}$ pour la reforme (iṣlāh) de ses serviteurs, Il les a distingués et les a réservés pour Son amour (li-widādihi). Dieu a dit: « Pour que Dieu distingue le mauvais du bon. » $(\text { Coran } 8,37)^{48}$.

45. Muntașiba, « solidement établit » doit être rapproché de manșub(a), le cas direct.

46. Les deux caractéristiques principales du hāl, l'indétermination grammaticale et le cas direct, qualifient symboliquement l'état (hāl) du connaissant devant Dieu. L'išāra repose sur la polyvalence sémantique de la notion de hậl. Le terme recouvre la signification de présent et d'état spirituel. Le fā'il se distingue du maf́ūl par la différence de cas grammatical, marfū opposé à manșūb. Qušayrī voit dans le fait que, contrairement au sujet et à l'objet, le hāal soit toujours indéfini et au cas direct, un dépassement de la dualité entre fā́il et maf́ūl, dépassement déjà anticipé dans le paragraphe précédent. L'état des hommes de Dieu est aussi indéterminé (nakira), dans le sens qu'ils fuient la célébrité, ce qu'on va retrouver dans le paragraphe suivant consacré au tamyīz. Qušayrī renvoie à la notion de tawriya, « ambiguïté », mais littéralement « le fait de cacher » son propre degré spirituel. Le paragraphe s'achève sur une citation du Coran 2, 273, faisant allusion à ceux qui cachent leur pauvreté par leur retenue, en s'interdisant de demander l'aumône. Qušayrī commente ce verset en mettant aussi en valeur l'effacement de l'âme :

« Dieu le Très-Haut a dit : « L'ignorant les croit riches à cause de leur retenue (ta'affuf) ». Celui qui est doué de clairvoyance ne rencontrera jamais de difficultés dans aucun de ses états. Reconnais-les, ô Muhammad, par leur marque (simma'), une marque qui ne paraît pas à la vue ordinaire (al-basar) mais que seule la clairvoyance (al-bașîra) peut saisir. [...] «Tu les reconnaitras à leur aspect » : leur cœurs se réjouissent dans le mépris de leurs âmes individuelles [... ]. » (Lațāif, vol. 1, p. 126.)

47. Jeu de mots sur nașaba, établir et manșūb, le cas direct du spécificatif.

48. Le complément de spécification (tamyiz) établit une relation entre deux noms d'une phrase, de sorte que le deuxième terme fait fonction de spécification restrictive au sens propre du premier, en soi vague et indéfini. Sur le plan formel, la spécification est reconnaissable par le cas direct et indéterminé (manșūb nakira). Qušayrī voit dans la spécification ce par quoi les hommes de Dieu se distinguent dans leur relation spéciale avec les créatures (dont ils sont les guides et les maîtres) et également avec le Créateur, qui les a réservés pour son amour. L'auteur utilise la portée sémantique du verbe mayyaza, dont le mașdar a pris le sens technique de tamyīz. Qušayrī revient au sens originel, celui de la distinction. Le facteur principal par lequel s'actualise cette distinction du vrai et du faux est la science. Ce rôle de la spécification est exprimé à partir d'une régle grammaticale : la spécification vient nécessairement à compléter le sens de la proposition, car sans elle, cette dernière ne serait pas parfaitement claire. De la même façon, la dernière catégorie de connaissants parachève les formes possibles de connaissance. L'idée de distinction repose tant sur le sens grammatical que sur le sens spirituel. Cette polyvalence, qui permet le mécanisme de l'išăra, est possible parce les deux disciplines (grammaire et tașawwuf) tirent du Coran un concept qui aboutit à un terme technique. Qušayrī cite l'acte de tamyīz dans le verset 38 de la sourate Al-Anfäl comme une action divin. L'action des hommes de la voie est donc ramenée à la volonté de Dieu qui fait jaillir le bien dans se monde en montrant le mal. Ainsi la méthode spirituelle des maîtres de la Voie se modèle sur cet acte divin. La spécification correspond à une élection au sein des croyants. Dans les Lața'if al-ishārāt, Qušayrī ne centre pas son commentaire sur la notion de tamyīz, mais sur la différence entre ce qu'est bon (tayyib) et ce qui est mauvais (habìt). Dieu est l'agent de cette distinction, et on retrouve la notion de iṣlāh, comme dans le Naḥw al-qulüb: 
L'APPOSITION (al-badal).

L'apposition (al-badal, lit. substitution) est de quatre sortes :

La substitution du tout par le tout (badal al-kull min al-kull) : il s'agit de la substitution des connaissants (al-'ārifīn), qui ont tout abandonné pour recevoir tout en échange. "Ce Jour-là il y aura des visages lumineux / contemplant leur Seigneur » (Coran 75, 22-23).

Les cœurs des connaisseurs ont des yeux qui voient ce que les regards ordinaires ne perçoivent pas,

Et des ailes, qui volent sans plumes vers le monde intérieur (malakūt) du Seigneur des Mondes.

La substitution d'une partie (badal al-ba'ḍ) :c'est la substitution des dévots (al-ābidinn), ils ont remplacé les désobéissances par les obéissances, et les plaisirs par les actes d'adoration. «Ceux-là Dieu substituera à leurs fautes des œuvres méritoires » (Coran 25, 70).

La substitution d'inclusion (badal al-ištimāl) : c'est la substitution de l'élite, dont les actions ont englobé à la fois espoir et crainte. Ils ont reçu ce qu'ils espéraient et ont été mis à l'abri de ce qu'ils craignaient. " N'est-il pas vrai que les saints de Dieu ne connaissent ni la peur ni l'affliction?»(Coran 10,62)

La substitution de l'erreur (badal al-ġalat) : c'est la substitution de ceux qui s'écartent de Dieu; ils ont vendu leur part de proximité pour des jouissances immédiates. «Quel mauvais échange pour les iniques! » (Coran 18, 50). ${ }^{49}$

\footnotetext{
«Mauvais est ce que Dieu n'a pas reformé, et bon est ce que Dieu a rétablit. Mauvais est ce que la Loi a décrété tel à cause de son caractère répugnant et corrompant, tandis que le bon est témoin de la science grâce à sa beauté et sa pureté. Ainsi le mécréant est dit mauvais, le croyant noble. Mauvais est ce qui distrait de Dieu, bon ce qui fait parvenir à Lui. Mauvais est ce que l'homme prend et dépense pour le plaisir de son âme, bon c'est ce qui est dépensé pour son Seigneur. » (Lațā’if, vol. 1, p. 393-394.)
}

49. Le terme "apposition" ne rende pas entièrement le sens des relations exprimé par le badal. Il s'agit plutôt d'une véritable substitution, là où le badal peut englober la signification et la fonction syntaxique du mubdal minhu selon une échelle de degrés : pleinement, partiellement, d'une façon « inclusive » ou « corrective ». Cette différence se manifeste dans la construction du badal, qui dans certains cas nécessite d'un pronom suffixe de rappel qui renvoie au mubdal minhu. Qušayrī présente le badal comme un principe de la Voie. Il propose quatre formes d'échange, selon un ordre hiérarchique, même si le badal ištimāl pose des problèmes, en sortant apparemment de cette échelle des valeurs. Dans ce sens, le badal al-kull devient symbole du remplacement advenu dans le cœur des connaissants (al'ärifin) qui ont tout abandonné pour recevoir en échange la totalité des choses et de la connaissance. Ce prototype de soufi est notamment celui de certains personnages comme Ibrāhīm b. Adham ou al-Fudayl, le premier un prince du Khorasan et le deuxième un riche bandit étant devenus de grands saints. Que cette catégorie de spirituels occupe dans la doctrine de Qušayrī la place la plus élevée est évidente lorsque qu'on lit le verset coranique qu'il leur attribue : il s'agit du plus haut degré de réalisation spirituelle, la contemplation de Dieu dans le Paradis. Dans les Lațā’if, le sens de nāzira - est compris comme «[visages] lumineux parce que plongés dans la contemplation de leur Seigneur [wa-hiya mushriqa li-annaha nāzira ay rā'iya Allāh] » (Lațāifi, vol. 3, p. 360). Le deuxième substitution est représenté par une autre catégorie d'hommes, celles des les dévots (al-ābidin). L'échange se situe au niveau de l'action et non de la contemplation. Le verset coranique qui, selon Qušayrī, décrit cette catégorie spirituelle est mis, dans les Latāàif, en relation avec l'idée de retour a Dieu (tawba) et pardon ( $\dot{g} u f r a ̄ n)$ :

«Ceux-là Dieu substituera à leurs fautes des œuvres méritoires (Coran 25, 70) : Dieu leur accorde la réussite à la place de l'échec. Il est dit aussi qu'il transformera leurs fautes en œuvres méritoires parce Il leur a pardonnés et Il a rendu lumineux leur retour à Lui » (Lațāifif, vol. 2, p. 394.) 


\section{L'ADJECTIF ÉPITHÈTE (al-na't).}

L'adjectif épithète est en accord parfait avec le nom qu'il suit et l'adjectif qualificatif est en accord parfait avec ce qu'il qualifie. De la même façon, les actions du serviteur ne le quittent jamais. Ainsi ce que lui arrive de bien ou de mal le suivra ${ }^{50}$.

\section{LA COORDINATION (hurūfal-'ațf).}

Les particules de coordination rattachent le dernier [élément] au premier. Les gens du sens allusif sont parvenus à Dieu par [Sa] bienveillance ('atff) à leur égard et [Sa] grâce subtile (lutff) envers eux, pour les rattacher aux gens de Sa proximité et les faire rejoindre Son parti ${ }^{51}$.

\section{LA CORROBORATION (al-tawkīd).}

La corroboration (tawkīd) est la réalisation spirituelle (al-tahqīq) ${ }^{52}$. Les gens de l'élite ont confirmé leur foi par la reconnaissance de la vérité (al-tașdìq) et leur engagement avec Dieu par le renforcement de leur lien avec Lui (tawtīq). Ils ont redoublé d'efforts pour persévérer sur la voie (šammarū fì mulāzama al-tarīq) ${ }^{53}$.

\footnotetext{
Le badal ištimāl, comme on l'a dit, sort apparemment de cette gradation de l'« échange » entre le bas monde et l'au-delà. A cette catégorie d'hommes, Qušayrī attribue le verset coranique sur les Saints, les proches de Dieu. Ce qui dans la Risāla est le propre des dévots (al-ubbād), est ici attribué à l'élite des croyants (al-qawm) : l'alternance de l'espoir et de la crainte, du désir et de la peur. La dernière forme d'apposition, le badal al-galaț, dans son sens grammatical, signifie que le badal annule le sens du mubdal minhu. L’allusion perçue par Qušayrī concerne ceux qui ont renoncé à l'au-delà pour la vie présente. Le verset de la sourate « La caverne » (Coran 18, 50), dans son ensemble concerne la désobéissance d'Iblīs :

«Et lorsque Nous enjoignîmes aux anges de se prosterner devant Adam, ils se prosternent sauf Iblīs qui était un djinn et qui se déroba à l'ordre de son Seigneur. Le prendrez-vous, lui et sa postérité, pour alliés en dehors de Moi alors qu'ils sont vos ennemis? Quelle détestable substitution pour les iniques!»
}

50. Ce bref paragraphe est centré sur le rapport étroit entre les actions de l'homme et son devenir. Les œuvres sont comparées à l'adjectif épithète, qui dans la syntaxe suit totalement l'objet qualifié. Cet accord, au niveau grammatical, implique le genre, le nombre, la détermination et le cas. Dans le Nahw al-qulūb, toutes ces déterminations syntaxiques ont un équivalent intérieur, dans le miroir de la grammaire des cœurs. Le na't et le wașf reflètent les actions du serviteur : elles suivent son essence et ne se séparent jamais de lui. On retrouve donc dans ce passage la pédagogie spirituelle de Qušayrī qui exige du murīd une unité d'être et d'action. Le passage à l'allusion est possible parce que le terme arabe pour « adjectif qualificatif », wașf, signifie aussi « qualité ». Le processus de l'išāra ici comme dans des autres passages du Naḥw al-qulūb est basé sur le sens étymologique de la notion grammaticale.

51. Qušayrī base son išära sur le retour au sens propre du mot 'ațf. Dans la grammaire des cœurs, les deux éléments reliés sont Dieu et son serviteur. Cette union (wușūl) est possible grâce à une forme de 'at $f$ spécial : l'amour de Dieu, son 'ațf (inclination, affection, sollicitude) envers ses créatures. Le verbe 'ațafa comprend les deux sens : 'ațafa ilā signifie « rattaché à »; 'ațafa 'alā, comme dans le texte de Qušayrī, «éprouver de la compassion pour ». Ce passage nous montre que dans la doctrine de Qušayrī, le wușūl est possible seulement comme don divin et non comme fruit des œuvres. Il est une conséquence de ce 'ațf et s'accompagne du lutf.

52. Tahqiq au sens grammatical signifie la réalisation de l'acte.

53. Dans le texte de Qušayrī, le principe du tawkīd est ramené à son sens étymologique de confirmation et de renforcement. Il s'agit toujours d'une relation entre deux éléments d'une phrase, et de la confirmation de l'un par l'autre. Dans le sens allusif que lui attribue l'auteur, le tawkìd devient la réalisation spirituelle. 
LES PARTICULES DU CAS INDIRECT (hurūf al-jarr).

Les particules du cas indirect «abaissent » (tahfidu) les noms. Lorsque que les êtres réalisés (al-muhaqqiqūn) ont pris connaissance que les choses sont par Dieu, venant de Lui et y retournant, ils abaissent leurs âmes par humilité envers Dieu et trouvent leur fierté en se rattachant à Dieu. C'est eux que Dieu a élus pour sa proximité et les a fait rejoindre Son parti. ${ }^{54}$ Nous demandons à Dieu l'Immense que Il nous compte parmi eux et nous rattache à eux. Il est Généreux, doué de grâce subtile, Longanime, donnant sans cesse, Bienfaiteur, plein de grâces, Magnanime, Miséricordieux, recevant le repentir, vers Lui est le retour et le refuge.

UN POÈME SUR LA GRAMMAIRE ${ }^{55}$.

La grammaire des cœurs est merveilleuse,

élévation ( $r a f$ ), abaissement (hafd), établissement (nașb)

Dans la marque de l'élévation ('alāmat al-raf') il y a

Esprit, intimité et proximité

Et dans les particules de l'abaissement (ahruf al-hafḍ)

Tristesse, contraction, voilement

Lâme est une particule ayant un sens

Qu'il est recommandé de supprimer.

Le complément d'état (al-hal) érige (yanșubu)

Ce que l'homme ne peut acquérir ${ }^{56}$,

Ça c'est la vraie grammaire, et non ce

qu'a dit 'Uțmān.

Une faute de langue est permise,

Mais la faute du cœur est péché,

Et la plus détestable des fautes pour moi

C'est orgueil, vanité et suffisance.

54. Ce dernier chapitre nous montre le sommet de la réalisation spirituelle, propre des êtres réalisés, al-muhaqqiqūn. On voit aussi que Qušayrī utilise la terminologie de Kufa et de Bașra, lorsqu'il emploie les notions de hafd et ğarr. «L'abaissement » auquel sont soumis les mots dans la grammaire ordinaire, devient dans la grammaire des cœurs l'image du tawādu', l'humilité devant Dieu. L'élément qui génère cette attitude est une prise de conscience centrale dans le cheminement spirituel : l'unité des choses en Dieu, seul et unique vrai agent de la réalité. Parmi les particules du cas indirect, Qušayrī se contente de citer explicitement l'annexion (idăfa $a$ ). Le fait d'être « lié » à Dieu, génère l'état de hafḍ de l'âme individuelle, de même que l'annexion grammaticale cause le cas oblique dans le terme annexé. Cette catégorie d'êtres sont ceux que Dieu a élevés au degré de sa proximité : il faut remarquer qu'il s'agit d'une proximité supra-temporelle. Qušayrī ne dit pas qu'ils seront, dans le Paradis, dans cette proximité : mais qu'ils le sont déjà dans cette vie, dans un rapport mutuel entre l'abaissement et l'élévation.

55. L'éditeur du texte signale que, à la marge du titre, dans un des manuscrits utilisés pour la rédaction de l'édition critique du Naḥw al-qulūb, on trouve les vers suivants. T. Ivàny suggère qu'ils ont été ajoutés par un copiste du manuscrit.

56. Allusion au fait que le hăl, dans son sens spirituel, est un don, alors que les maqāmāt sont des acquisitions (makāsib). 